EPJ Web of Conferences 71, 00065 (2014)

DOI: $10.1051 /$ epjconf / 20147100065

(C) Owned by the authors, published by EDP Sciences, 2014

\title{
Doubly Heavy Tetraquarks and Baryons
}

\author{
Marek Karliner ${ }^{1, a}$ \\ ${ }^{1}$ Raymond and Beverly Sackler School of Physics and Astronomy \\ Tel Aviv University, Tel Aviv, Israel
}

\begin{abstract}
During the last three years strong experimental evidence from B and charm factories has been accumulating for the existence of exotic hadronic quarkonia, narrow resonances which cannot be made from a quark and an antiquark. Their masses and decay modes show that they contain a heavy quark-antiquark pair, but their quantum numbers are such that they must also contain a light quark-antiquark pair. The theoretical challenge has been to determine the nature of these resonances. The main possibilities are that they are either "genuine tetraquarks", i.e. two quarks and two antiquarks within one confinement volume, or "hadronic molecules" of two heavy-light mesons. In the last few months there as been more and more evidence in favor of the latter. I discuss the experimental data and its interpretation and provide fairly precise predictions for masses and quantum numbers of the additional exotic states which are naturally expected in the molecular picture but have yet to be observed. In addition, I provide arguments in favor of the existence of an even more exotic state - a hypothetical deuteron-like bound state of two heavy baryons. I also consider "baryon-like" states $Q Q^{\prime} \bar{q} \bar{q}^{\prime}$, which if found will be direct evidence not just for near-threshold binding of two heavy mesons, but for genuine tetraquarks with novel color networks. I stress the importance of experimental search for doubly-heavy baryons in this context.
\end{abstract}

\section{First observation of manifestly exotic hadrons}

In late 2007 the Belle Collaboration reported [1] anomalously large partial widths of $\Upsilon(5 S) \rightarrow \Upsilon(2 S)$ and $\Upsilon(5 S) \rightarrow \Upsilon(1 S)$, two orders of magnitude larger than the analogous decays of $\Upsilon(3 S)$. Soon afterward Harry Lipkin and I proposed [2] that a four-quark exotic resonance $[b \bar{b} u \bar{d}]$ might mediate these decays through the cascade $\Upsilon(m S) \rightarrow[b \bar{b} u \bar{d}] \pi^{-} \rightarrow \Upsilon(n S) \pi^{+} \pi^{-}$. We suggested looking for the $[b \bar{b} u \bar{d}]$ resonance in these decays as peaks in the invariant mass of $\Upsilon(1 S) \pi$ or $\Upsilon(2 S) \pi$ systems. More recently Belle collaboration confirmed this prediction, announcing $[3,4]$ the observation of two charged bottomonium-like resonances $Z_{b}$ as narrow structures in $\pi^{ \pm} \Upsilon(n S)(n=1,2,3)$ and $\pi^{ \pm} h_{b}(m P)$ $(m=1,2)\left(h_{b}\right.$-s are spin-singlet, $P$-wave bottomonia with $\left.J=1\right)$ mass spectra that are produced in association with a single charged pion in $\Upsilon(5 S)$ decays.

Since these states decay into bottomonium and a charged pion, they must contain both a $\bar{b} b$ heavy quark-antiquark pair and a $\bar{d} u$ light quark-antiquark pair. Thus their minimal quark content is $\bar{b} b \bar{d} u$. They are manifestly exotic. Their discovery by Belle was the first time such manifestly exotic hadron resonances have been unambiguously observed experimentally.

ae-mail: marek@proton.tau.ac.il 


\title{
Possibility of Exotic States in the Upsilon system
}

\author{
Marek Karliner ${ }^{a *}$ \\ and \\ Harry J. Lipkin ${ }^{a, b \dagger}$
}

\begin{abstract}
Recent data from Belle show unusually large partial widths $\Upsilon(5 S) \rightarrow$ $\Upsilon(1 S) \pi^{+} \pi^{-}$and $\Upsilon(5 S) \rightarrow \Upsilon(2 S) \pi^{+} \pi^{-}$. The $Z(4430)$ narrow resonance also reported by Belle in $\psi^{\prime} \pi^{+}$spectrum has the properties expected of a $\bar{c} c u \bar{d}$ charged isovector tetraquark $T_{\bar{c} c}^{ \pm}$. The analogous state $T_{\bar{b} b}^{ \pm}$in the bottom sector might mediate anomalously large cascade decays in the Upsilon system, $\Upsilon(m S) \rightarrow T_{\bar{b} b}^{ \pm} \pi^{\mp} \rightarrow \Upsilon(n S) \pi^{+} \pi^{-}$, with a tetraquark-pion intermediate state. We suggest looking for the $\bar{b} b u \bar{d}$ tetraquark in these decays as peaks in the invariant mass of $\Upsilon(1 S) \pi$ or $\Upsilon(2 S) \pi$ systems. The $\bar{b} b u \bar{s}$ tetraquark can appear in the observed decays $\Upsilon(5 S) \rightarrow \Upsilon(1 S) K^{+} K^{-}$ as a peak in the invariant mass of $\Upsilon(1 S) K$ system. We review the model showing that these tetraquarks are below the two heavy meson threshold, but respectively above the $\Upsilon \pi \pi$ and $\Upsilon K \bar{K}$ thresholds.
\end{abstract}

Figure 1. First page of our paper [2] proposing a four-quark exotic intermediate state $b \bar{b} u \bar{d}$ as an explanation of the anomalously large rate partial widths of $\Upsilon(5 S) \rightarrow \Upsilon(2 S)$ and $\Upsilon(5 S) \rightarrow \Upsilon(1 S)$.

The measured masses of the two structures averaged over the five final states are $M_{1}=10608.4 \pm 2.0 \mathrm{MeV}, M_{2}=10653.2 \pm 1.5 \mathrm{MeV}$, both with a width of about $15 \mathrm{MeV}$. Interestingly enough, the two masses $M_{1}$ and $M_{2}$ are about $3 \mathrm{MeV}$ above the respective $B^{*} \bar{B}$ and $B^{*} \bar{B}^{*}$ thresholds.

\subsection{Interpretation as deuteron-like quasi-bound states of two heavy mesons}

The most interesting theoretical question is what are these states? Their quantum numbers are those of a $\bar{b} b \bar{u} d$ tetraquark, but such quantum numbers can also be realized by a system consisting of $B^{*} \bar{B}$ and $B^{*} \bar{B}^{*}$ "hadronic molecules" loosely bound by pion exchange, as schematically shown in Figure 2.

The difference between these two possibilities is subtle, because they have the same quantum numbers and therefore in principle they can can mix with each other. The extent of the mixing depends on the overlap between the respective wave functions. By a "tetraquark" I mean a state where all four quarks are within the same "bag" or confinement volume, while by "hadronic molecule" I mean a state where there are two color-singlet heavy-light mesons attracting each other by exchange of pions and possibly other light mesons.

The proximity of the two resonances to the $B^{*} \bar{B}$ and $B^{*} \bar{B}^{*}$ thresholds strongly suggests a parallel with $X(3872)$, whose mass is almost exactly at the $D^{*} \bar{D}$ threshold.

It also provides strong support for the the possibility that these state indeed are deuteron-like "molecules" of two heavy mesons quasi-bound by pion exchange [6-8], as schematically shown in Figure 3. This is because it is very unlikely that two "genuine" tetraquarks just happened to sit at the respective two-meson thresholds. 


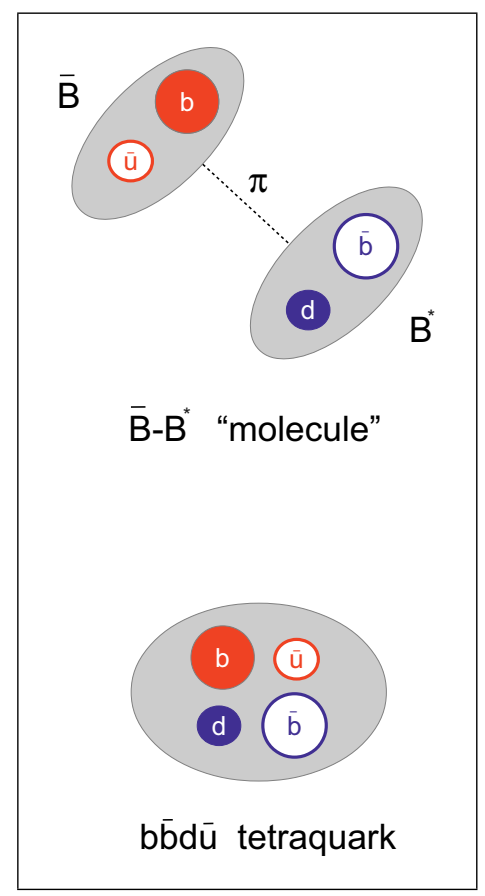

Figure 2. A schematic depiction of a $\bar{B}-B^{*}$ deuteron-like "hadronic molecule" vs. a $b \bar{b} d \bar{u}$ tetraquark. Note that, unlike in the "molecule", in the tetraquark configuration the $b \bar{u}$ and $d \bar{b}$ diquarks in general won't be color singlets.
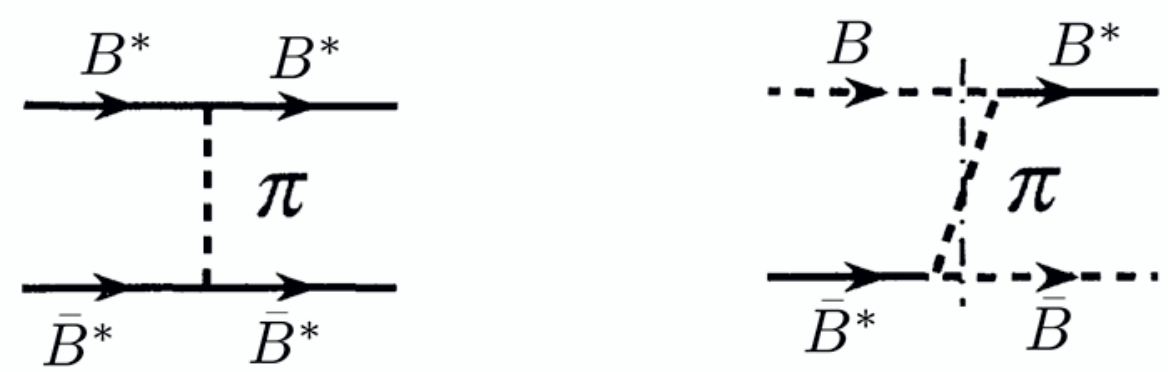

Figure 3. Diagrams contributing to $B \bar{B}^{*}$ and $B^{*} \bar{B}^{*}$ binding through pion exchange. Analogous diagrams contribute to $D \bar{D}^{*}$ and $D^{*} \bar{D}^{*}$ binding, modulo the caveat that $D^{*} \rightarrow D \pi$, while $B^{*} \rightarrow B \pi$ is kinematically forbidden, as discussed in the text.

The attraction due to $\pi$ exchange is 3 times weaker in the $I=1$ channel than in the $I=0$ channel. Consequently, in the charm system the $I=1$ state is expected to be well above the $D^{*} \bar{D}$ threshold and the $I=0 \quad X(3872)$ is at the threshold. ${ }^{1}$ In the bottom system the attraction due to $\pi$ exchange is essentially the same, but the kinetic energy is much smaller by a factor of $\sim m(B) / m(D) \approx 2.8$. Therefore the net binding is much stronger than in the charm system.

\footnotetext{
${ }^{1}$ For simplicity we treat $X(3872)$ as an isoscalar, since it has no charged partners, and we ignore here the issue of isospin breaking in its decays. A more refined treatment results in the same conclusions.
} 
The recently discovered manifestly exotic charged resonances are surprisingly narrow. This is the case in both $\bar{b} b$ systems [3, 4] - and in the exotic charmonium, namely the remarkable peak $Z_{c}(3900)$ at $3899.0 \pm 3.6 \pm 4.9 \mathrm{MeV}$ with $\Gamma=46 \pm 10 \pm 20 \mathrm{MeV}$ reported by BESIII [5].

The relatively slow decay of these exotic resonances implies that the dominant configuration of the $\bar{Q} Q \bar{q} q$ four-body system is not that of a low-lying $\bar{Q} Q$ quarkonium and pion(s). The latter have a much lower energy than the respective two-meson thresholds $\bar{M} M^{*}$ and $\bar{M}^{*} M^{*},(M=D, B)$, but do readily fall apart into $(\bar{Q} Q)$ and pion(s) and would result in very large decay widths. Thus we should view these systems as loosely bound states and/or near threshold resonances in the two heavy-meson system.

Such "molecular" states, $\bar{D} D^{*}$, etc., were introduced in Ref. [6]. They were later extensively discussed [7, 8] in analogy with the deuteron which binds via exchange of pions and other light mesons, and were referred to as "deusons". The key observation is that the coupling to the heavy mesons of the light mesons exchanged $\left(\pi, \rho\right.$, etc.) becomes universal and independent of $M_{Q}$ for $M_{Q} \rightarrow \infty$, and so does the resulting potential in any given $J^{C P}$ and isospin channel. In this limit the kinetic energy $\sim p^{2} /\left(M_{Q}\right)$ vanishes, and the two heavy mesons bind with a binding energy $\sim$ the maximal depth of the attractive meson-exchange potential.

For a long time it was an important open question whether these consideration apply in the real world with large but finite masses of the $D$ and $B$ mesons. The recent experimental results of Belle [3, 4] and BESIII [5], together with theoretical analysis in Refs. [9] and [10] strongly indicate that such exotic states do exist - some were already found and more are predicted below.

Due to parity conservation, the pion cannot be exchanged in the $\bar{M} M$ system, but it does contribute in the $\bar{M} M^{*}$ and $\bar{M}^{*} M^{*}$ channels. This fact provides additional support to the molecular interpretation, because resonances have been observed close to $\bar{D} D^{*}, \bar{D}^{*} D^{*}, \bar{B} B^{*}$ and $\bar{B}^{*} B^{*}$ thresholds, but no resonances have been seen at $\bar{D} D$ nor at $\bar{B} B$ thresholds.

The $\vec{\tau}_{1} \cdot \vec{\tau}_{s}$ isospin nature of the exchange implies that the the binding is 3 times stronger in the isoscalar channel. It was estimated $[9,10]$ that in the bottomonium system this difference in the binding potentials raises the $I=1$ exotics well above the $I=0$ exotics. In the charmonium system this splitting is expected to be slightly smaller, because the $\bar{D} D^{*} / \bar{D}^{*} D^{*}$ states are larger than $\bar{B} B^{*} / \bar{B}^{*} B^{*}$. This is because the reduced mass in the $\bar{B} B^{*}$ system is approximately 2.5 times larger than in the $\bar{D} D^{*}$ system. On the other hand, the net attractive potential due to the light mesons exchanged between the heavy-light mesons is approximately the same, since $m_{c}, m_{b} \gg \Lambda_{Q C D}$. As usual in quantum mechanics, for a given potential the radius of a bound state or a resonance gets smaller when the reduced mass grows, so the $\bar{D} D^{*}$ states are larger than the $\bar{B} B^{*}$ states. Because of this difference in size the attraction in both $I=0$ and $I=1$ charmonium channels is expected to be somewhat smaller. In addition, Ref. [11] suggested that the asymptotic coupling between the two heavy mesons and a pion, $g_{M M^{*} \pi}$ for $m_{Q} \rightarrow \infty$ is approached from below, so that $g_{B B^{*} \pi}>g_{D D^{*} \pi}$.

\subsection{Prediction of additional related exotic states}

Since the quarks are heavy, we can treat their kinetic energy as a perturbation depending linearly on a parameter inversely proportional to $\mu_{\text {red }}$, the reduced mass of the two meson system, which scales like the mass of the heavy quark [12], with the hamiltonian $H=a \cdot p^{2}+V$, where $a=1 / \mu_{\text {red }} \sim 1 / m_{Q}$. We can use the existing data in order to make a very rough estimate of the isovector binding potential in the $m_{Q} \rightarrow \infty$ limit. We have two data points: $Z_{c}(3900)$ at $a(D)$ is approximately $27 \mathrm{MeV}$ above $\bar{D} D^{*}$ threshold and $Z_{b}(10610)$ at $a(B)$ is approximately $3 \mathrm{MeV}$ above $\bar{B} B^{*}$ threshold. Linear extrapolation to $a=0$ yields $E_{b}^{I=1}(a=0) \approx-11.7 \mathrm{MeV}$. In view of the convexity of the binding energy in $a$, the actual binding energy is likely to slightly exceed this linear extrapolation. 
We can then use this result for the isovector channel to estimate the $\bar{B} B^{*}$ binding in the isoscalar channel. Assuming that the isoscalar binding energy in the $m_{Q} \rightarrow \infty$ limit is 3 times larger than for the isovector, we have $E_{b}^{I=0}(a=0) \approx 3 \cdot(-11.7)=-35 \mathrm{MeV}$. The state $X(3872)$ is at $\bar{D} D^{*}$ threshold, providing an additional data point of $E_{b}^{I=0}(a(D)) \approx 0$ in the isoscalar channel. Linear extrapolation to $a(B)$ yields approximately $-20 \mathrm{MeV}$ as the $\bar{B} B^{*}$ binding energy in the isoscalar channel.

The upshot is that the newly discovered $Z_{c}(3900)$ isovector resonance confirms and refines the estimates in $[9,10]$ for the mass of the putative $\bar{B} B^{*}$ isoscalar bound state. This immediately lead to several predictions [12]:

a) two $I=0$ narrow resonances $X_{b}$ in the bottomonium system, about $23 \mathrm{MeV}$ below $Z_{b}(106010)$ and $Z_{b}(10650)$, i.e. about $20 \mathrm{MeV}$ below the corresponding $\bar{B} B^{*}$ and $\bar{B}^{*} B^{*}$ thresholds;

b) an $I=1$ resonance above $\bar{D}^{*} D^{*}$ threshold;

c) an $I=0$ resonance near $\bar{D}^{*} D^{*}$ threshold.

The $X_{b}$ states can most likely be observed through the decays $X_{b} \rightarrow \Upsilon \omega$ or $X_{b} \rightarrow \chi_{b} \pi \pi$. Unlike incorrectly stated in [12], they cannot decay to $\Upsilon \pi \pi$. The latter decays are prevented by $G$-parity conservation.[13] The observed decay $X(3872) \rightarrow J / \pi \pi$ is only possible because isospin is strongly broken between $D^{+}$and $D^{0}$, and because $X(3872)$ is at the $\bar{D} D^{*}$ threshold. In contradistinction, in the bottomonium system isospin is almost perfectly conserved. Thus the null result in CMS search [14] for $X_{b} \rightarrow \Upsilon(1 S) \pi^{+} \pi^{-}$does not tell us if $X_{b}$ exists.

Quite recently the BESIII collaboration reported observation in $e^{+} e^{-} \rightarrow\left(D^{*} \bar{D}^{*}\right)^{ \pm} \pi^{\mp}$ of what looks just like (b) above, namely a new charmonium-like charged resonance $Z_{c}(4025)$, slightly above the $\bar{D}^{*} D^{*}$ threshold, at $\sqrt{s}=(4026.3 \pm 2.6 \pm 3.7) \mathrm{MeV}$, with width of $24.8 \pm 5.6 \pm 7.7 \mathrm{MeV}$ [15]. Shortly afterward, BESIII reported observation of another charged charmoniumlike structure $Z_{c}(4020)$ in $e^{+} e^{-} \rightarrow \pi^{+} \pi^{-} h_{c}$ at $(4022.9 \pm 0.8 \pm 2.7) \mathrm{MeV}$ and width of $7.9 \pm 2.7 \pm 2.6 \mathrm{MeV}$. At this time it is not yet clear if these are two independent resonances, or two observations of the same object at slightly different masses, possibly due to systematic effects associated with the two observation channels.

Figure 4 provides a concise summary of the experimental information about the masses of doublyheavy exotics observed so far, together with our predictions for the masses of additional states, as discussed above. Figure 5 shows the observed and predicted decay channels of these states.

It is somewhat puzzling that, unlike $Z_{c}^{+}(3900), Z_{c}^{+}(4020 / 4026)$ has not been seen in the $J / 4 \pi^{+}$ mode. Moreover, one notes that $Z_{c}^{+}(4020 / 4026)$ is somewhat closer to the $\bar{D}^{*} D^{*}$ threshold than our prediction. It will be interesting to identify the reasons for this small difference. The two main possibilities are (a) details of the experimental analysis; (b) a possible difference between the $\bar{B}^{*} B^{*}$ and $\bar{D}^{*} D^{*}$ attractive pion-exchange potentials. Such a difference might perhaps be due to the fact that $m\left(B^{*}\right)-[m(B)+m(\pi)] \approx-94 \mathrm{MeV}$, while $m\left(D^{*}\right)-[m(D)+m(\pi)] \approx 0^{ \pm}$, depending on the $D^{*}$ and $\pi$ charges, affecting energy denominators in virtual pion emission.

\subsection{A $\left(\Sigma_{b}^{+} \Sigma_{b}^{-}\right)$beauteron dibaryon?}

The discovery of the $Z_{b}$-s and their interpretation as quasi-bound $B^{*} \bar{B}$ and $B^{*} \bar{B}^{*}$, raises an interesting possibility of a strongly bound $\Sigma_{b}^{+} \Sigma_{b}^{-}$deuteron-like state, a beauteron [17]. The $\Sigma_{b}$ is about $500 \mathrm{MeV}$ heavier than $B^{*}$. The $\Sigma_{b} \Sigma_{b}$ kinetic energy is therefore significantly smaller than that of $B \bar{B}^{*}$ or $B^{*} \bar{B}^{*}$. Moreover, $\Sigma_{b}$ with $I=1$ couples more strongly to pions than $B$ and $B^{*}$ with $I=\frac{1}{2}$. The opposite electric charges of $\Sigma_{b}^{+}$and $\Sigma_{b}^{-}$provide additional 2-3 MeV of binding energy. The heavy dibaryon bound state might be sufficiently long-lived to be observed experimentally. A possible decay mode of 
EPJ Web of Conferences

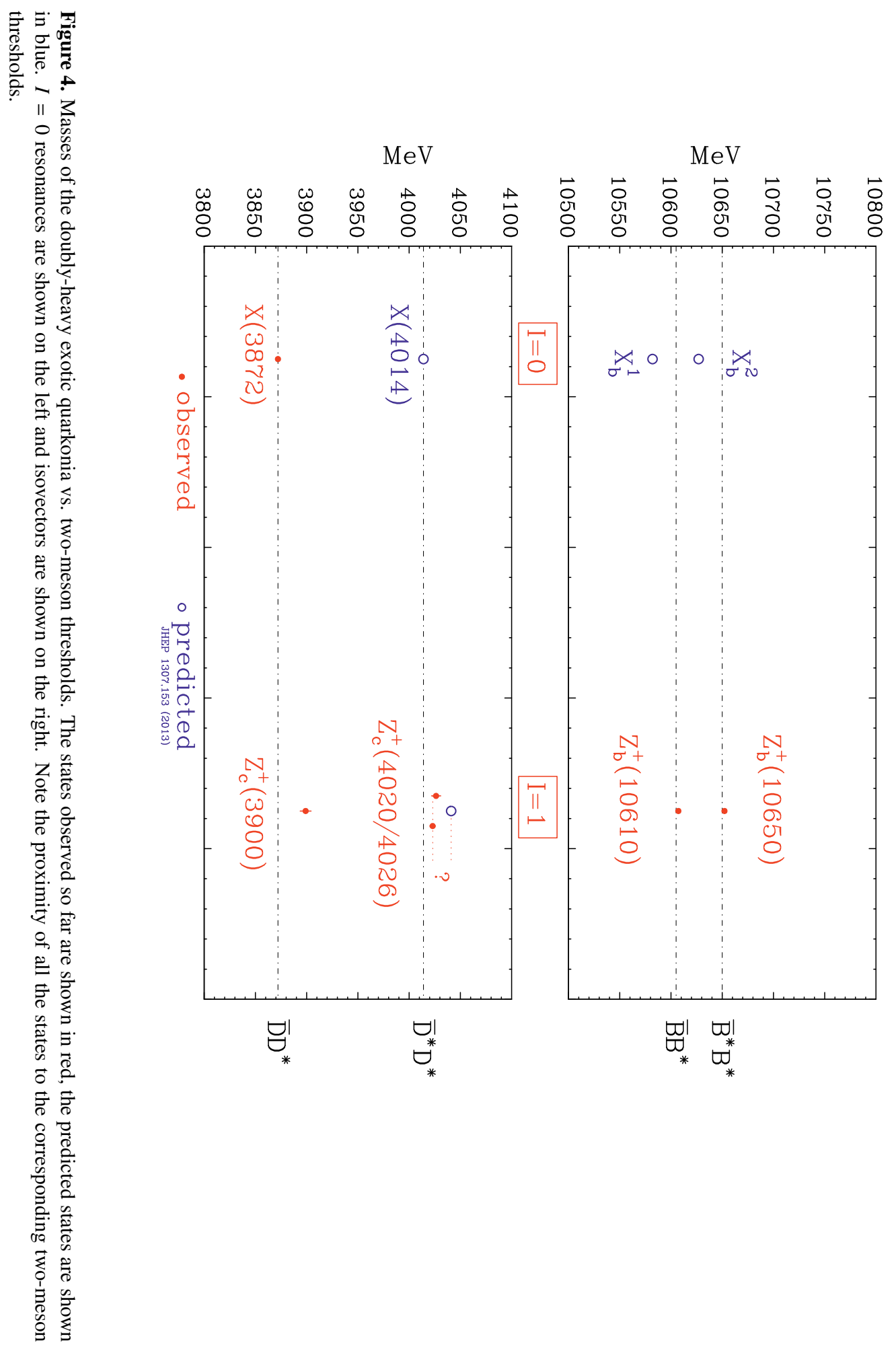


ICNFP 2013

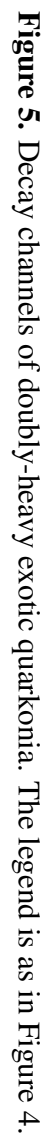

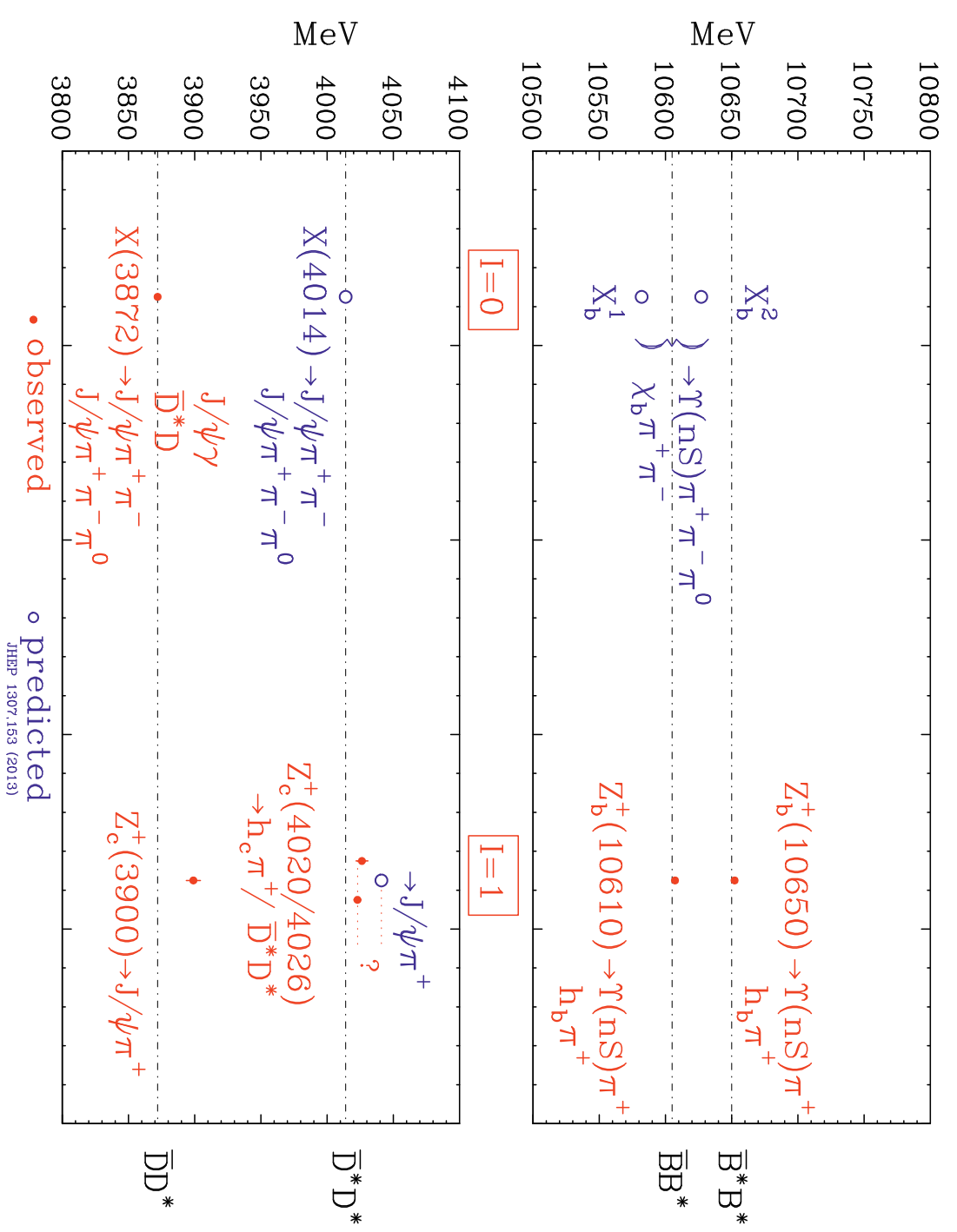


the beauteron is $\left(\Sigma_{b}^{+} \Sigma_{b}^{-}\right) \rightarrow \Lambda_{b} \Lambda_{b} \pi^{+} \pi^{-}$, which might be observable in LHCb. It should also be seen in lattice QCD.

\section{$2 Q Q \bar{q} \bar{q}$ tetraquarks}

The quark content of the exotic resonances observed so far is $\bar{Q} Q \bar{q} q$. A very different type of exotics are the $Q Q \bar{q}_{1} \bar{q}_{2}$ tetraquarks (TQ-s) [18-21]. If such states do exist, producing and discovering even the lightest $c c \bar{u} \bar{d}$ is an extraordinary challenge. One needs to produce two $\bar{Q} Q$ pairs and then rearrange them, so as to form $Q Q$ and $\bar{Q} \bar{Q}$ diquarks, rather than the more favorable configuration of two $\bar{Q} Q$ and color singlets. Then the $Q Q$ diquark needs to pick up a $\bar{u} \bar{d}$ light diquark, rather than a $q$, to make a $Q Q q$ baryon, suppressing the production rate of these TQ-s below the rate of $Q Q q$ doubly-heavy baryons production which themselves are quite hard to make, as discussed below.

A small ray of hope comes from the observation of the doubly-heavy $B_{c}=(\bar{b} c)$ mesons [22, 23], suggesting that simultaneous production of $\bar{b} b$ and $\bar{c} c$ pairs which are close to each other in space and in rapidity and can coalesce to form doubly-heavy hadrons is not too rare. For example CDF reported [23] $108 \pm 15$ candidate events in the $B_{c}^{ \pm} \rightarrow J / \psi \pi^{ \pm}$channel. This is an encouraging sign for the prospects of producing and observing the $c c q$ and $b c s$ baryons and hopefully also the $c c \bar{u} \bar{d}$ TQ. ATLAS and CMS and especially LHCb have the best chance of discovering these states.

If the new TQ lies below the two heavy meson threshold, it will be stable under the strong interaction and will decay only weakly. ${ }^{2}$ A discovery of such a stable exotic hadron would be a spectacular finding indeed. As we discuss below, if these tetraquarks lay above threshold, they still may manifest themselves as narrow $D D^{*}$ resonances.

The following theoretical considerations are relevant for the last point above. First, in clear contradistinction to the states discussed earlier, the new TQ-s are unlikely to be molecular. This is because both heavy mesons contain light antiquarks, rather than a $\bar{q}$ in one and a $q$ in the other, causing the $\omega$ and $\rho$ exchange to be repulsive, rather than attractive. With no bound $\bar{D} D^{*}$ states, one might expect the new $D D^{*}$ states to lay higher and manifest themselves as broad resonances, at best.

This bleak picture will change dramatically if the $Q Q \bar{u} \bar{d}$ TQ is not a simple molecular state, but instead a connected color network, consisting of a $Q Q$ diquark coupled to a $\mathbf{3}_{c}^{*}$, with a matching $\bar{u} \bar{d}$ diquark coupled to a $\mathbf{3}_{c}$. The $Q Q$ diquark will then be bound by the large Coulombic interaction $O\left(\alpha_{s}^{2} M_{Q}\right)$, as discussed below. Finding such a novel color network would be particularly exciting [20],[25]. With some poetic license, we can compare it to the discovery of the $C_{60}$ buckyballs.

The Coulombic binding of the $Q Q$ diquark,

$$
E_{b}(Q Q)=\frac{1}{2} \cdot \frac{1}{2} \cdot \frac{1}{2} \alpha_{s}^{2} M_{Q}=\frac{1}{8} \alpha_{s}^{2} M_{Q}
$$

is half as strong as that of a color-singlet $\bar{Q} Q$. Another $\frac{1}{2}$ factor reflects the need to use the reduced mass, and the $\frac{1}{2} \alpha_{s}^{2} M_{Q}$ is the standard hydrogen binding formula. Thus the heavy diquark $Q Q$ becomes infinitely deeply bound as $M_{Q} \rightarrow \infty$. For $m_{c} \sim 1.6 \mathrm{GeV}$ and using $1 / 2$ for the relevant $\alpha_{s}$, the Coulombic binding $\sim 50 \mathrm{MeV}$ is moderate. However, the $\frac{1}{2}$ ratio of QCD Coulomb interaction in the diquark, versus its strength in the $\bar{Q} Q$ color singlet system likely extends to all QCD interactions, including the confining stringy potential [26].

If indeed the $c c \bar{u} \bar{d}$ TQ is mostly described by the new color network, then its decay into $D D^{*}$ will be suppressed by the required rearrangement of the color.

In view of the above discussion, we now focus on the question whether the lowest state of the new color network is below or near the $D D^{*}$ threshold.

\footnotetext{
${ }^{2}$ A statement slightly modified if the TQ mass is above the $2 m_{D}$ threshold, as it can then first decay through an exceedingly narrow EM decay $D^{*} \rightarrow D^{0} \gamma[11,24]$, followed by the weak decays of the the $D$-s [20].
} 


\section{Doubly-heavy baryons}

From the point of view of QCD there is nothing exotic about baryons containing two heavy quarks ( $b$ or $c$, generically denoted by $Q$ ) and one light quark ( $u$ or $d$, generically denoted by $q$ ). Heavy quarks decay only by weak interaction, with a characteristic lifetime orders of magnitude larger than the typical QCD timescale, so from the point of view of strong interactions the $Q Q q$ baryons are stable, just like protons, neutrons and hyperons. Thus these doubly-heavy baryons must exist.

As discussed above in the context of $Q Q \bar{q} \bar{q}$ tetraquarks, producing and discovering such states is a significant experimental challenge. One has to produce two $\bar{Q} Q$ pairs which need to rearrange themselves, so as to form $Q Q$ and $\bar{Q} \bar{Q}$ diquarks, rather than the more favorable configuration of two $\bar{Q} Q$ color singlets. Then the $Q Q$ diquark needs to pick up a an light quark $q$, to make a $Q Q q$ baryon.

There are interesting parallels between the $Q Q \bar{q} \bar{q}$ TQ-s and doubly-heavy baryons $Q Q q$. In both types of systems there is a light color triplet - a quark or an anti-diquark - bound to a heavy diquark. Because of this similarity, experimental observation of doubly-heavy baryons is very important not just in its own right, but as source of extremely valuable information for deducing the properties of the more exotic $Q Q \bar{q} \bar{q}$ tetraquarks. Such deduction can carried out just as it was done for $b$-baryons.

In the last few years it became possible to accurately predict at the level of 2-3 MeV the masses of heavy baryons containing the $b$-quark: $\Sigma_{b}(b q q), \Xi_{b}(b s q)$ and $\Omega_{b}(b s s)$ [27-29], as shown in Figure 6.

These predictions used as input the masses of the $B, B_{s}, D$ and $D_{s}$ mesons, together with the masses of the corresponding $c$-baryons $\Sigma_{c}(c q q), \Xi_{c}(c s q)$ and $\Omega_{c}(c s s)$.

An analogous approach to the masses of the $Q Q \bar{q} \bar{q}$, suggested the relation

$$
m(c c \bar{u} \bar{d})=m\left(\Xi_{c c u}\right)+m\left(\Lambda_{c}\right)-m\left(D^{0}\right)-\frac{1}{4}\left[m\left(D^{*}\right)-m(D)\right]
$$

designed to optimally match interactions on both sides [20],[12]. To date, only the SELEX experiment at Fermilab reported doubly charmed $c c d$ and $c c u$ baryons with mass $\sim 3520 \mathrm{MeV}$ [30] - a result not confirmed by other experiments [31,32]. Substituting this mass in eq. (2) as a placeholder in a "proof of concept" estimate yields $M(\operatorname{cc} \bar{u} \bar{d}) \sim 3900 \mathrm{MeV}$, which is just $30 \mathrm{MeV}$ above the $D^{*} D$ threshold. One should keep in mind that, as implied by eq. (1), the binding in $(b b \bar{q} \bar{q})$ is expected to be significantly stronger than in $(c c \bar{q} \bar{q})$. In any case, reliable experimental information on $Q Q q$ baryon masses is clearly essential for settling the issue.

Hadrons containing two $b$ quarks, such as double-bottom baryons $b b q$ or $b \bar{b} q \bar{q}$ and $b b \bar{q} \bar{q}$ tetraquarks have a unique and a spectacular decay mode with two $J / \psi$-s in the final state. It is mediated by both $b$ quarks decaying via $b \rightarrow \bar{c} c s \rightarrow J / \psi s$ and yields

$$
(b b q) \rightarrow J / \psi J / \psi(s s q) \rightarrow J / \psi J / \psi \Xi
$$

and

$$
(b \bar{b} q \bar{q}) \rightarrow J / \psi J / \psi(\bar{s} s \bar{q} q) \rightarrow J / \psi J / \psi K \bar{K}
$$

as well as

$$
(b b \bar{q} \bar{q}) \rightarrow J / \psi J / \psi(s s \bar{q} \bar{q}) \rightarrow J / \psi J / \psi \bar{K} \bar{K},
$$

etc., with all final state hadrons coming from the same vertex. This unique signature is however hampered by a very low rate, expected for such a process, especially if one uses dimuons to identify the $J / \psi$-s. It is both a challenge and an opportunity for LHCb [25].

Once the double bottom baryon is identified experimentally through (3) or via another channel and its mass is measured, one will immediately be able to estimate the mass of the corresponding 
EPJ Web of Conferences

\section{b-baryons spectrum - TH predictions vs EXP}

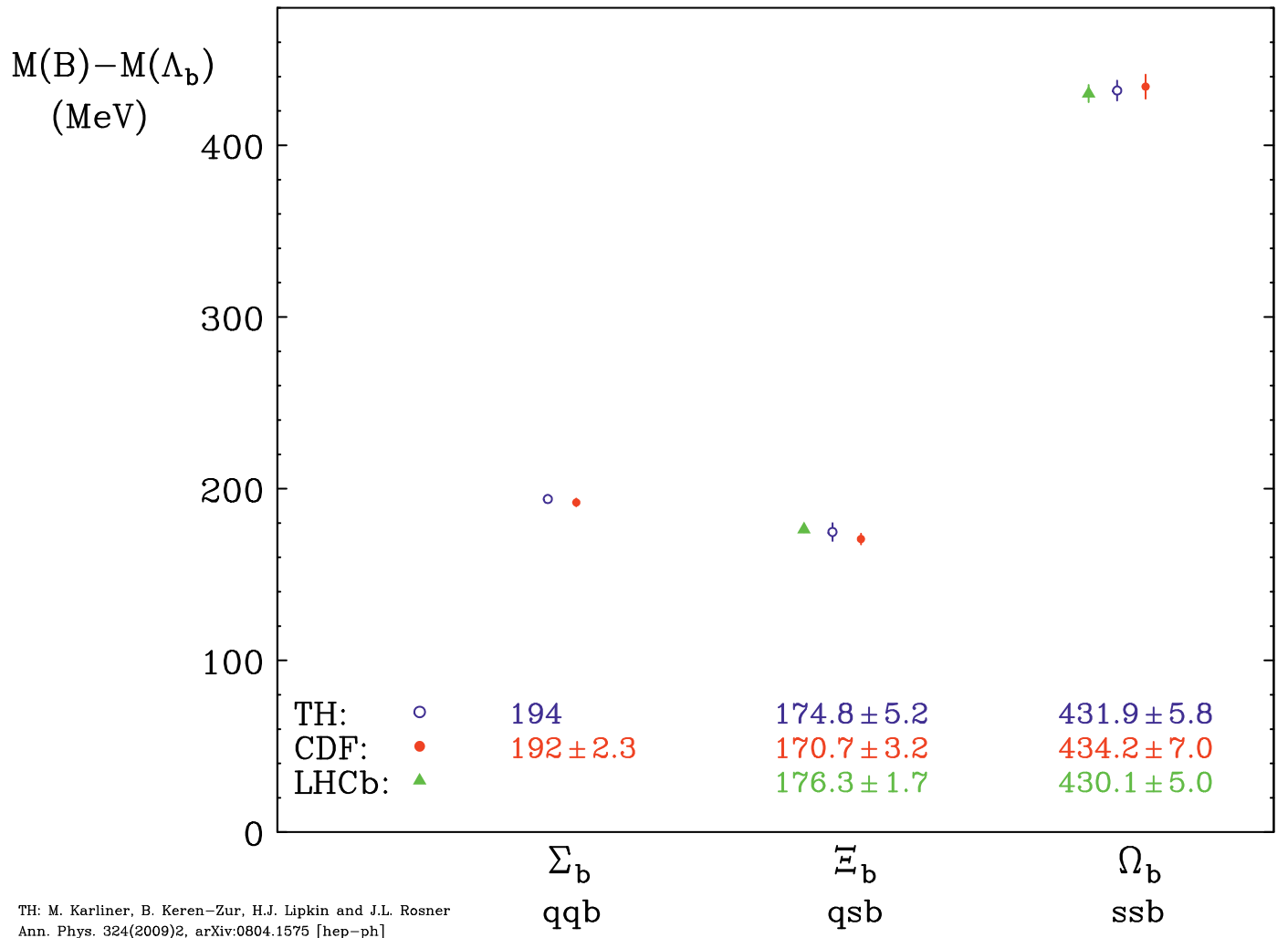

Figure 6. Comparison of theoretical predictions [27-29] and experiment for masses of $b$-baryons.

tetraquark through the $b$-quark analogue of eq. (2) and check whether or not it is below the two $B$ meson threshold:

$$
m(b b \bar{u} \bar{d})=m\left(\Xi_{b b u}\right)+m\left(\Lambda_{b}\right)-m\left(B^{0}\right)-\frac{1}{4}\left[m\left(B^{*}\right)-m(B)\right]
$$

\section{References}

[1] K. F. Chen et al. [Belle Collaboration], Phys. Rev. Lett. 100, 112001 (2008) [arXiv:0710.2577 [hep-ex]].

[2] M. Karliner and H. J. Lipkin, arXiv:0802.0649 [hep-ph].

[3] I. Adachi et al., Belle Coll., arXiv:1105.4583 [hep-ex].

[4] A. Bondar et al. [Belle Collaboration], Phys. Rev. Lett. 108, 122001 (2012) [arXiv:1110.2251 [hep-ex]].

[5] M. Ablikim et al. [ BESIII Collaboration], arXiv:1303.5949 [hep-ex].

[6] M. B. Voloshin and L. B. Okun, JETP Lett. 23, 333 (1976) [Pisma Zh. Eksp. Teor. Fiz. 23, 369 (1976)]. 
[7] N. A. Tornqvist, Phys. Rev. Lett. 67, 556 (1991); N. A. Tornqvist, Z. Phys. C 61, 525 (1994) [hep-ph/9310247].

[8] N. A. Tornqvist, Phys. Lett. B 590, 209 (2004) [hep-ph/0402237].

[9] M. Karliner, H. J. Lipkin, N. A. Tornqvist, arXiv:1109.3472 [hep-ph].

[10] M. Karliner, H. J. Lipkin, N. A. Tornqvist, Nucl. Phys. Proc. Suppl. 225-227, 102 (2012).

[11] S. Nussinov, Phys. Lett. B 418, 383 (1998).

[12] M. Karliner and S. Nussinov, JHEP 1307, 153 (2013) [arXiv:1304.0345 [hep-ph]].

[13] I thank Roman Mizuk for discussion on this point.

[14] S. Chatrchyan et al. [CMS Collaboration], arXiv:1309.0250 [hep-ex].

[15] M. Ablikim et al. [BESIII Collaboration], arXiv:1308.2760 [hep-ex].

[16] M. Ablikim et al. [BESIII Collaboration], arXiv:1309.1896 [hep-ex].

[17] M. Karliner, H.J. Lipkin, N.A. Törnqvist, unpublished and [arXiv:1109.3472].

[18] J. P. Ader, J. M. Richard and P. Taxil, Phys. Rev. D 25, 2370 (1982).

[19] A. V. Manohar and M. B. Wise, Nucl. Phys. B 399, 17 (1993) [hep-ph/9212236].

[20] B. A. Gelman, S. Nussinov, Phys. Lett. B 551, 296 (2003) [hep-ph/0209095].

[21] Y. Frishman and M. Karliner, JHEP 1308, 096 (2013) [arXiv:1305.6457 [hep-ph]].

[22] K. Ackerstaff et al. [OPAL Collaboration], Phys. Lett. B 420, 157 (1998) [hep-ex/9801026];

F. Abe et al. [CDF Collaboration], Phys. Rev. Lett. 81, 2432 (1998) [hep-ex/9805034]; A. Abulencia et al. [CDF Collaboration], Phys. Rev. Lett. 96, 082002 (2006) [hep-ex/0505076]; V. M. Abazov et al. [D0 Collaboration], Phys. Rev. Lett. 101, 012001 (2008) [arXiv:0802.4258 [hep-ex]];

[23] T. Aaltonen et al. [CDF Collaboration], Phys. Rev. Lett. 100, 182002 (2008) [arXiv:0712.1506 [hep-ex]].

[24] J. Beringer et al. [Particle Data Group Collaboration], Phys. Rev. D 86, 010001 (2012).

[25] M. Karliner, H. J. Lipkin, Phys. Lett. B 638, 221 (2006) [hep-ph/0601193].

[26] S. Nussinov and M. A. Lampert, Phys. Rept. 362, 193 (2002) [hep-ph/9911532]

[27] M. Karliner and H.J. Lipkin, arXiv:hep-ph/0307243; condensed version in Phys. Lett. B575 (2003) 249.

[28] M. Karliner, B. Keren-Zur, H. J. Lipkin and J. L. Rosner, arXiv:0706.2163 [hep-ph].

[29] M. Karliner, B. Keren-Zur, H. J. Lipkin and J. L. Rosner, Annals Phys. 324, 2 (2009) [arXiv:0804.1575 [hep-ph]].

[30] M. Mattson et al. [SELEX Collaboration], Phys. Rev. Lett. 89, 112001 (2002) [hep-ex/0208014];

A. Ocherashvili et al. [SELEX Collaboration], Phys. Lett. B 628, 18 (2005) [hep-ex/0406033].

[31] B. Aubert et al. [BABAR Collaboration], Phys. Rev. D 74, 011103 (2006) [hep-ex/0605075].

[32] R. Chistov et al. [BELLE Collaboration], Phys. Rev. Lett. 97, 162001 (2006) [hep-ex/0606051]. 